\title{
Porous-Coated Metaphyseal Sleeves in Revision Total Knee Arthroplasty: Midterm Results
}

\author{
Giacomo Stefani $^{1}{ }^{10}$ Valerio Mattiuzzo ${ }^{1}$ Greta Prestini ${ }^{1}$ Carolina Civitenga ${ }^{1}$ Roberto Calafiore ${ }^{2}$ \\ Francesco Traverso ${ }^{3}$
}

${ }^{1}$ Istituto Clinico "Città di Brescia," Brescia, Italy

${ }^{2}$ Casa di cura "Città di Parma," Parma, Italy

${ }^{3}$ Istituto Humanitas, Rozzano, Milano, Italy

Joints 2019;7:135-140.
Address for correspondence Giacomo Stefani, MD, Istituto Clinico Città di Brescia, Via Gualla 15, Brescia 25128, Italy

(e-mail: trstefa@libero.it).

\begin{abstract}
Keywords

- knee

- total knee arthroplasty

- revision

- metaphyseal sleeves

Purpose The aim of this study was to evaluate the efficacy in terms of clinical results and radiographic findings of using metaphyseal sleeves in revision total knee arthroplasty (TKA), and to check if the use of sleeves without stems did not impair such results.

Methods In this retrospective study, 141 patients (143 knees) operated in the period 2008 to 2015 met the above-mentioned criteria and were invited to a medical examination including X-rays. A total of 121 knees were available for the study (44 in the group without stems and 77 in the group with stems). Mean follow-up was 63 months for the stemless group and 89 for the group with stems. Knee Society Score (KSS) (objective knee score) and Western Ontario and McMaster Universities Osteoarthritis Index (WOMAC) were used as outcome scores and compared with baseline values, range of motion (ROM) was also compared with preop value and $\mathrm{X}$-rays were also examined and compared with immediate postop X-rays to check signs of loosening and radiolucent lines, if any, and bone ingrowth. Satisfaction of the patients was also investigated using a linear scale from 1 to 10.

Results KSS improved from 34 to 81 postop (39 to 81 in the stemless group) $(p<0.01$ ), while WOMAC from $82 \%$ preop to $39 \%$ postop (76 to $37 \%$ in the stemless group) $(p<0.01)$. Forty-six patients were satisfied, 20 partially satisfied, and 11 unsatisfied (respectively $25-14-5$ in the stemless group). ROM improved from 89 degrees preop to 99 degrees postop (93 to 98 degrees in the stemless group). $\mathrm{X}$-rays showed no loosening of the implant, radiolucent lines in 8 patients, and bone ingrowth in 113 out of 121 patients.

Conclusion In this midterm follow-up study, we found a significant improvement in clinical results compared with preop values. We found no difference between the two groups (with and without stems) thus suggesting that the use of stemless sleeves does not impair results in revision TKA.

Level of Evidence Therapeutic case series, level IV.
\end{abstract}

received

October 20, 2019 accepted after revision April 18, 2021 published online June 14, 2021
DOI https://doi.org/ $10.1055 / \mathrm{s}-0041-1730377$. ISSN 2282-4324. (c) 2021. The Author(s).

This is an open access article published by Thieme under the terms of the Creative Commons Attribution-NonDerivative-NonCommercial-License, permitting copying and reproduction so long as the original work is given appropriate credit. Contents may not be used for commercial purposes, or adapted, remixed, transformed or built upon. (https://creativecommons.org/ licenses/by-nc-nd/4.0/)

Georg Thieme Verlag KG, Rüdigerstraße 14, 70469 Stuttgart, Germany 


\section{Introduction}

In revision total knee arthroplasty (TKA) a good fixation of the implant and bone management are the most critical issues that a surgeon must face. ${ }^{1}$ Usually, fixation has been achieved with intramedullary stems either cemented or press-fit; leading to possible stem pain and use of off-set stems to adapt to tibial and femoral anatomy. ${ }^{2-4}$ Managing bone loss could require use of cement, bone chips, and structural bone allograft but each of these techniques has several problems, in particular structural allografts require a technically demanding procedure with possible late resorption of the graft itself and disease transmission. ${ }^{5-8}$ Porous tantalum cones are also effectively used but they have two interfaces, one with bone and one with implant, leading to possible weakness of the implant itself ${ }^{9,10}$; moreover, surgical technique is difficult and surgeon experience is mandatory.

In the last years there has been interest in obtaining fixation in the metaphyseal region in an attempt to improve construct stability while managing bone loss at the same time. ${ }^{11}$ Quite a few authors have been using metaphyseal sleeves in revision cases as support or alternative to stems, reporting satisfactory early and midterm results. ${ }^{12-20}$

Tip pain after revision TKA is well known and documented ${ }^{21}$ and the use of metaphyseal sleeves may be an effective alternative to stem fixation. Moreover, the shape of the sleeve may also contribute to manage bone loss both in the tibia and on the femoral side.

The purpose of our study was to evaluate the effectiveness of sleeves in implant fixation and bone management in revision TKA. We also investigated if the use of sleeves without stems did not impair such fixation and survival.

\section{Methods}

From December 2008 until December 2015, 141 patients (143 knees) were revised for a failed TKA in our department, with the use of metaphyseal sleeves, with or without stems, for implant fixation. The selection of patients included all the cases in which a sleeve was used. Eighteen patients ( 19 knees) in which only stems were used are not included in the study and comparison was not done given the very different sample size. In all cases, a SIGMA TC3 DePuy revision implant was used with a posterior stabilized insert in 123 knees and a TC3 (varus-valgus constrained [VVC] constraint) insert in 18 knees. In 51 of these knees cementless metaphyseal sleeves without stem either on the tibia or femoral side or in both, were used. In three of these cases aTC3 insert was used. In two cases (in the sleeves with stem group) a hinge was used. This is not a consecutive case series because at the beginning we were still using sleeves with stems because we were not so confident in the use of sleeves without stem yet. The uncommon cases in which we use stems are those in which the contact area of the sleeve with the metaphyseal bone is less than $70 \%$ on average and when the quality of bone is very poor.

Reasons for revisions were: aseptic loosening 61 (42\%), periprosthetic joint infection 46 (32\%), instability $11(8 \%)$, fracture $6(4 \%)$, poliwear $4(3 \%)$, arthrofibrosis $4(3 \%)$, and other causes $11(8 \%)$. In $92(64 \%)$ patients it was the first revision, in 33 (23\%) it was the second, and in 18 (13\%) it was the third or more. Note that $101(71 \%)$ patients were female and $42(29 \%)$ male and the average age at the time of revision was 73 years ( $\min 46$, max 87).

Anderson Orthopedic Research Institute (AORI) classification of defects prior to revision surgery was: AORI 3 in 21 femur and 16 tibia, AORI 2A-2B in 98 femur and 102 tibia, and AORI 1 in 24 femur and 25 tibia.

A total of 120 metaphyseal sleeves were used in the femur and 138 in the tibia (respectively 41 and 46 in the stemless group). In the group with stems, 44 tibial and 71 femoral stems were used.

\section{Surgical Technique}

Failed TKA was removed. Forty-six (32\%) patients (from the infection and the arthrofibrosis group) underwent an anterior tibia tubercle (ATT) osteotomy to gain better exposure during surgery, that was then fixed either with screws, metal wire, or both (-Fig. 1A-C).

We usually perform ATT osteotomy during second stage revision of a periprosthetic joint infection and in revision for stiff TKA; in four cases we performed a quadriceps snip. The tibia canal is identified and reamed to 10 to $12 \mathrm{~mm}$ width and approximately $150 \mathrm{~mm}$ depth using also a conical reamer, then preparation of the tibia for the sleeve is done using sequential broaching with broaches that must have a narrow stem at the tip to maintain the correct direction in the canal. Good fixation of the broach at the level that we consider optimal for obtaining a correct joint line, according to preop planning, is achieved when it is possible to lift the leg off the table using the broaching jig. We then cut proximal tibia and quite often this can be done using the broach itself as a support base for cutting.

We measure with block spacers extension and flexion gap, than we proceed to femur preparation in a similar way, checking the correct rotation of the femoral component. Once we have found the correct direction of the broach and we have the feeling of good rotation stability we may remove the guidance stem, and this allows slight flexion of the broach to compensate the flexion gap (if necessary). Position of the tibia tray was obtained keeping the center of the tibia tubercle as reference and femoral component with the balance technique at 90 degrees of flexion. Fixation of the final components with sleeves was done with cement on the baseplate only, being careful to avoid putting it on the sleeve. Cement with antibiotics was used in all cases.

Surgical complication included 13 anterior linear tibia fractures and 6 on the metaphyseal anterior cortex of the femur, all of them associated with sleeve preparation and/or insertion. In three of the femoral fractures we performed a cerclage with metal wire. They all healed without further treatment.

Patients were allowed weight bearing as tolerated and encouraged to reach a good range of motion (ROM) starting from the first postop day. Patients with an ATT osteotomy wore a brace for 6 weeks, locked in extension, weight bearing as tolerated, and continuous passive motion up to 90 degrees in 30 days. 

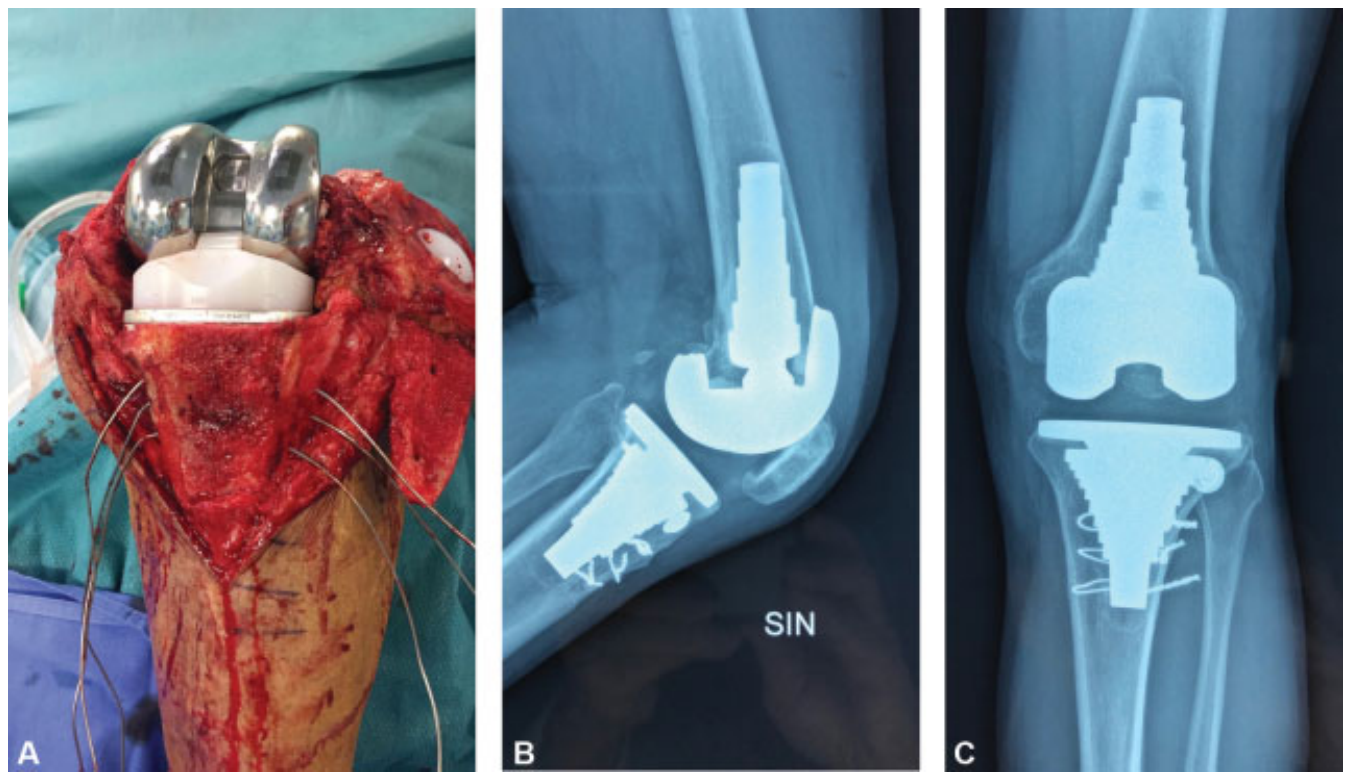

Fig. 1 (A) Revision total knee arthroplasty (TKA) for periprosthetic joint infection. Osteotomy of anterior tibial tuberosity is done, to get better exposure, but stability of the sleeve and of the implant is not impaired. Fixation can be done with metal wires, screws, or both. (B, C) X-ray of the same case 62 months postop. Good clinical and radiological result. No signs of recurrence of infection neither mobilization of the implant. Note in - Fig. 1 B flexion of the femoral sleeve with anterior contact with cortical bone. This maneuver allowed us to equalize flexion gap with extension gap but no pain was experienced by the patient in this case and in similar situations.

\section{Outcome Measurements}

Patients were evaluated before surgery and at follow-up according to the objective Knee Society Score (KSS) and the Western Ontario and McMaster Universities Osteoarthritis Index (WOMAC) score. A subjective satisfaction score was also obtained on a linear scale from 1 to 10 and rated as satisfied (score 7-10), partially satisfied (4-6), and unsatisfied (1-3). Standard A-P and L-L radiographs were also taken at follow-up and compared with postop radiographs to see if bone ingrowth had occurred, according to the criteria of Engh et $\mathrm{al}^{22}$ and if any radiolucent line had appeared.

\section{Statistical Analysis}

A paired Student's $t$-test was used for statistical analysis to compare outcome scores with baseline values. Significance was set at $p$-value $<0.05$.

\section{Results}

Out of 141 patients (143 knees) in which we used metaphyseal sleeves in revision TKA either in the tibia or femoral side or in both, 121 patients ( 121 knees) were available for the study. Six had to be revised: 3 for infection, 1 for instability, 1 for periprosthetic femoral fracture, and 1 for patellar problems, and were excluded from the study, because failure was not related to sleeves, 3 died, 4 were not traceable, and 9 declined.

Average follow-up was 89 months (min 42, max 102) overall and 63 months ( $\min 42, \max 86)$ in the stemless group.

The mean KSS score improved from 34 preop to 81 postop (39 to 81 in the stemless group) $(p<0.01)$, mean WOMAC from $82 \%$ preop to $39 \%$ postop (76 to $37 \%$ in the stemless group) $(p<0.01)$.
ROM improved from 89 degrees preop to 99 degrees postop (93-98 degrees, respectively) in the stemless group but it was not significant. In the stemmed group, 46 patients (60\%) were satisfied with the result, 20 patients (26\%) were partially satisfied, and 11 patients (14\%) were unsatisfied (respectively 25-14-5 in the stemless group). Among these, 9 patients belonged to the infection group and 1 to the arthrofibrosis group that did not improve in ROM after surgery.

X-rays showed an overall good fixation of prosthesis, according to the criteria of Engh et al. In 8 patients radiolucent lines ( 3 femoral, 5 tibia) could be seen around the sleeves, 6 of those without clinical symptoms. The two symptomatic patients are under surveillance. Radiological cortical contact of the sleeves was found in 18 patients, 12 in the femur, on the anterior cortex, and 6 in the tibia but no pain was related to it. In one case of sleeves with stem, in the tibial side, the patient experienced tip pain due to lateral migration of the stem that might require revision. All the ATT osteotomies showed good fixation without secondary displacement of the tibia tubercle.

\section{Discussion}

The purpose of this study was to evaluate the efficacy in terms of clinical result and radiographic findings of revision TKA in which metaphyseal sleeves were used for implant fixation. In fact, literature is now quite consistent in showing good midterm results of this procedure, but the further purpose of this study was to evaluate the efficacy of metaphyseal sleeves used without stems in revision TKA.

In recent years there has been a great interest in obtaining fixation in the metaphyseal region to improve construct stability while managing bone deficiency and avoiding diaphyseal fixation with long stems. ${ }^{11}$ Stem fixation has been used for 
a long time in revision TKA with satisfactory results, nevertheless some problems like pain at the end of the stem ${ }^{21}$ and difficulties in positioning the stem itself if the femoral and tibia canal were not straight ${ }^{2-4}$ have been described, even with offset design, limiting in some ways the efficacy of this procedure. Moreover, in many cases, the metaphyseal region has still fairly good bone quality and it seems reasonable to use it to fix the implant without the need of stem. This was documented by X-rays that showed an overall stable fixation of prosthesis without signs of loosening. Radiological bone ingrowth, comparing postop with follow-up X-rays was seen in 113 out of 121 knees, similar to the ratio showed by Dalury and Barrett. $^{12}$

Clinical evaluation using the objective KSS score and WOMAC functional score showed significant improvement from baseline values and patients' overall satisfaction (satisfied or partially satisfied) was reported in 105 patients, thus confirming the efficacy of the procedure. Among the 16 unsatisfied patients, 9 belonged to the infection group, 1 patient to the stiffness group that did not improve ROM after surgery, and 1 with tip pain. Nevertheless, further exams in this subgroup of 16 patients did not show any recurrence of infection. The use of sleeves is becoming popular and many authors $^{12-20}$ have shown positive results in revision TKA. Most of them, though, are using stems with sleeves for fixation, even if some authors ${ }^{13}$ report that in their study half of the tibia and femoral metaphyseal sleeves (40 and $66 \%$, respectively) were implanted without the use of a stem, and none of these have shown evidence of early loosening, thus confirming the efficacy of this procedure. Some are cementing the sleeves (55\% femoral and $72 \%$ tibial) showing good results, ${ }^{19}$ but this technique is very uncommon among European surgeons, being the porous-coated surface of the sleeve supposed to be used without cement.

Due to its shape, the peripheral contact area of the sleeve with the bone is very wide, and if a good fixation with the sleeve is obtained in the metaphyseal region, solid fixation of the implant is achieved. In accordance with zonal fixation concept, two zones should be required for a stable fixation in revision TKA. ${ }^{23}$ With the use of stems alone this is absolutely true, but with sleeves a good fixation in a large area can be achieved using zone 2 . In this case, fixation in zone 3 becomes less relevant, and thus stem size and percentage of canal-filling can be reduced or the use of stem can be even avoided, still finding no significant difference between stemless and stemmed augmentation. ${ }^{13,14}$ Moreover, in the femur the use of stem makes it difficult to flex the femoral component (even with offset stems, which are available but not easy to use either) and this maneuver is often helpful to compensate flexion gap in some cases. Without stem this maneuver is possible and easy to perform and up to 6 or 7 degrees of flexion can be achieved, thus filling up to $5 \mathrm{~mm}$ of flexion gap. The flexion of the component, though, has not caused any extension deficit in our series. Sometime, in these cases, the proximal tip of the sleeve is in contact with the anterior cortex of the femur, but no pain is related to that because loading of the sleeve is on the conic, porous-coated, area, far from the contact area. Nevertheless, stems are necessary during surgery to find

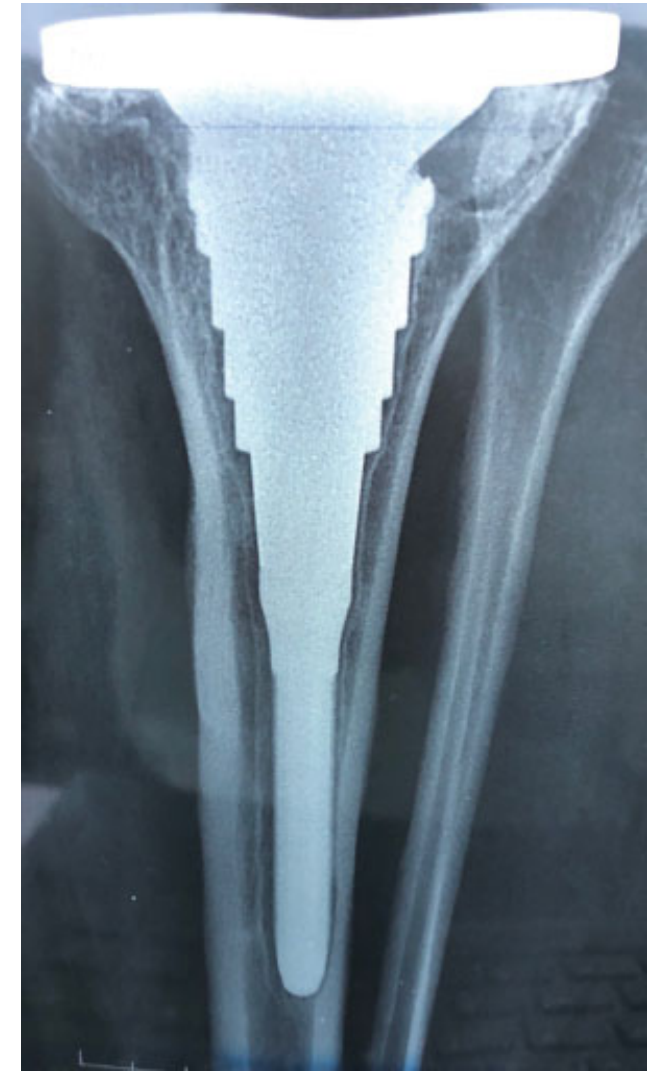

Fig. 2 Sleeve with stem at 81 months. Note lateral migration of stem with cortical contact and tip pain. We are planning to revise the patient, remove sleeve and stem, and use a larger sleeve only.

a correct alignment in the medullary canal and, at the beginning of a surgeon's learning curve, we believe that a narrow stem is acceptable, just to maintain a straight direction and final correct position of the implant, but the stem should have no contact with the diaphyseal cortical bone. In one early case, the use of a small sleeve, with a narrow stem, has caused a lateral migration of the stem with tibial pain (-Fig. 2). The patient is scheduled to have a re-revision with removal of sleeve and stem and use a larger sleeve for fixation. Our experience suggests that using larger sleeves to have a primary strong fixation may lead to the use of sleeves without stems.

In 46 patients (32\%), we performed an ATT osteotomy during revision, in all cases we were able, despite the sleeve, to make a stable fixation of the tuberosity either with metal wire, screws, or both. In the cases with fractures we had neither any serious problem during rehabilitation nor any secondary implant migration. ROM showed a very limited improvement after surgery: 89 to 99 degrees (93-98 degrees, respectively, in the stemless group). This value was not significant. Other authors ${ }^{14}$ showed in their series a significant improvement of ROM from 89 degrees preop to 114 degrees postop, but they had excluded septic revision from their study, while others ${ }^{12}$ showed worsening of ROM from 125 degrees preop to 115 degrees at final follow-up. We believe that the limited increase of ROM after surgery in our series depends on the fact that we included a large percentage 46 (32\%) of septic revisions in which we used a brace and allowed a limited ROM during the initial rehabilitation period. 

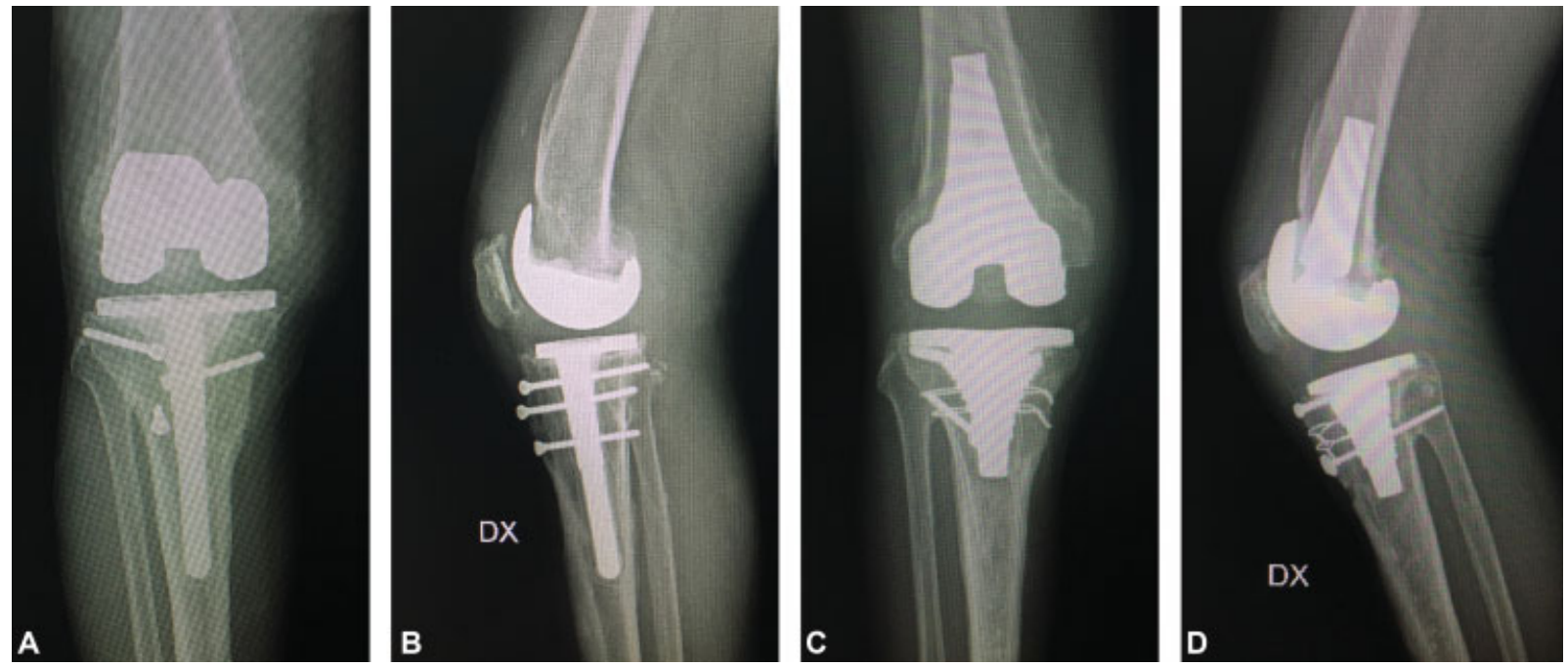

Fig. 3 (A, B) X-Ray at 8 years after a revision total knee arthroplasty (TKA), now with tip pain and stiffness. (C, D) Re-revision at 54 months. Good implant position, no pain. Note that despite the previous anterior tibia tubercle (ATT) osteotomy it was possible to perform the same procedure with unconstrained implant and good result.

From a biomechanical point of view, the fixation with sleeves, rather than with stems, is much closer to the joint line, thus making ligament balance easier, ${ }^{24}$ and allowing use of unconstrained implants that may have longer survivorship. ${ }^{25}$ In fact, only 2 hinges and 18 TC3 inserts (VVC constraint) were used ( - Fig. 3A-D). A great advantage in daily practice is that the use of sleeves provides bone defect management and implant fixation within the same surgical step.

We still use stems, preferably uncemented, when the sleeve has a contact surface with the metaphyseal bone that is less than $70 \%$ on the average or when the bone is of very poor quality. We have also to consider that the use of stemless sleeves is a money saving procedure comparing it with the use of sleeves with stems and knowing the economic impact of revision TKA in health care the use of less expensive procedures, once their efficacy is confirmed, is desirable.

There are few limitations in our study. This is a retrospective evaluation of a nonconsecutive case series, which implies a risk of selection bias. At the beginning we were not confident enough and we applied the procedure without stems to easy cases, then with experience we have learned to treat the great majority of revision TKA with this procedure. Today, approximately 90\% of all our revision TKAs are performed with stemless sleeves.

The relatively short follow-up time is another limitation; however, the results at a medium follow-up of 89 months ( 63 in the stemless group) are encouraging. On the other hand, some studies ${ }^{26-28}$ show that at least $50 \%$ of all re-revision occur in the first 2 to 3 years. So even if we could not make a survival analysis a tendency to longer survivorship is evident. Nevertheless, we are aware that an even longer follow-up is required to validate this procedure before a final assessment can be made.

\section{Conclusion}

In this retrospective study we investigated the effectiveness of sleeves in revision TKA and we confirmed that it is a safe procedure. We found a significant improvement in clinical results compared with baseline values and no sign of implant loosening. Results are not impaired by the use of stemless sleeves thus encouraging us in continuing with this procedure.

Conflict of Interest

G.S. is a paid consultant of DePuy Synthes.

\section{References}

1 Engh GA, Ammeen DJ. Bone loss with revision total knee arthroplasty: defect classification and alternatives for reconstruction. Instr Course Lect 1999;48:167-175

2 Fehring TK, Odum S, Olekson C, Griffin WL, Mason JB, McCoy TH. Stem fixation in revision total knee arthroplasty: a comparative analysis. Clin Orthop Relat Res 2003;(416):217-224

3 Whaley AL, Trousdale RT, Rand JA, Hanssen AD. Cemented longstem revision total knee arthroplasty. J Arthroplasty 2003;18(05): 592-599

4 Manopoulos P, Havet E, Pearce O, Lardanchet JF, Mertl P. Mid- to long-term results of revision total knee replacement using pressfit intramedullary stems with cemented femoral and tibial components. J Bone Joint Surg Br 2012;94(07):937-940

5 Whiteside LA. Morselized allografting in revision total knee arthroplasty. Orthopedics 1998;21(09):1041-1043

6 Dorr LD, Ranawat CS, Sculco TA, et al. Bone graft for tibial defects in total knee arthroplasty. Clin Orthop Relat Res 2001;(392):306-314

7 Bauman RD, Lewallen DG, Hanssen AD. Limitations of structural allograft in revision total knee arthroplasty. Clin Orthop Relat Res 2009;467(03):818-824

8 Sculco PK, Abdel MP, Hanssen AD, Lewallen DG. The management of bone loss in revision total knee arthroplasty: rebuild, reinforce, and augment. Bone Joint J 2016;98-B(1, Suppl A):120-124

9 Meneghini RM, Lewallen DG, Hanssen AD. Use of porous tantalum metaphyseal cones for severe tibial bone loss during revision total knee replacement. J Bone Joint Surg Am 2008;90(01):78-84

10 Kamath AF, Lewallen DG, Hanssen AD. Porous tantalum metaphyseal cones for severe tibial bone loss in revision knee arthroplasty: a five to nine-year follow-up. J Bone Joint Surg Am 2015;97(03):216-223

11 Haidukewych GJ, Hanssen A, Jones RD. Metaphyseal fixation in revision total knee arthroplasty: indications and techniques. J Am Acad Orthop Surg 2011;19(06):311-318 
12 Dalury DF, Barrett WP. The use of metaphyseal sleeves in revision total knee arthroplasty. Knee 2016;23(03):545-548

13 Bugler KE, Maheshwari R, Ahmed I, Brenkel IJ, Walmsley PJ. Metaphyseal sleeves for revision total knee arthroplasty: good short-term outcomes. J Arthroplasty 2015;30(11):1990-1994

14 Graichen H, Scior W, Strauch M. Direct, cementless, metaphysical fixation in knee revision arthroplasty with sleeves: short-term results. J Arthroplasty 2015;30(12):2256-2259

15 Barnett SL, Mayer RR, Gondusky JS, Choi L, Patel JJ, Gorab RS. Use of stepped porous titanium metaphyseal sleeves for tibial defects in revision total knee arthroplasty: short term results. J Arthroplasty 2014;29(06):1219-1224

16 Watters TS, Martin JR, Levy DL, Yang CC, Kim RH, Dennis DA. Porous-coated metaphyseal sleeves for severe femoral and tibial bone loss in revision TKA. J Arthroplasty 2017;32(11):3468-3473

17 Martin-Hernandez C, Floria-Arnal LJ, Muniesa-Herrero MP, et al Mid-term results for metaphyseal sleeves in revision knee surgery. Knee Surg Sports Traumatol Arthrosc 2017;25(12):3779-3785

18 Fedorka CJ, Chen AF, Pagnotto MR, Crosset LS, Klatt BA. Revision total knee arthroplasty with porous-coated metaphyseal sleeves provides radiographic ingrowth and stable fixation. Knee Surg Sports Traumatol Arthrosc 2018;26(05):1500-1505

19 Chalmers BP, Desy NM, Pagnano MW, Trousdale RT, Taunton MJ. Survivorship of metaphyseal sleeves in revision total knee arthroplasty. J Arthroplasty 2017;32(05):1565-1570

20 Zanirato A, Cavagnaro L, Basso M, Divano S, Felli L, Formica M. Metaphyseal sleeves in total knee arthroplasty revision: complications, clinical and radiological results. A systematic review of the literature. Arch Orthop Trauma Surg 2018;138(07): 993-1001

21 Barrack RL, Rorabeck C, Burt M, Sawhney J. Pain at the end of the stem after revision total knee arthroplasty. Clin Orthop Relat Res 1999;(367):216-225

22 Engh CA, Massin P, Suthers KE. Roentgenographic assessment of the biologic fixation of porous-surfaced femoral components. Clin Orthop Relat Res 1990;(257):107-128

23 Morgan-Jones R, Oussedik SI, Graichen H, Haddad FS. Zonal fixation in revision total knee arthroplasty. Bone Joint J 2015; 97-B(02):147-149

24 Bieger R, Huch K, Kocak S, Jung S, Reichel H, Kappe T. The influence of joint line restoration on the results of revision total knee arthroplasty: comparison between distance and ratio-methods. Arch Orthop Trauma Surg 2014;134(04):537-541

25 Shen C, Lichstein PM, Austin MS, Sharkey PF, Parvizi J. Revision knee arthroplasty for bone loss: choosing the right degree of constraint. J Arthroplasty 2014;29(01):127-131

26 Mortazavi SM, Molligan J, Austin MS, Purtill JJ, Hozack WJ, Parvizi J. Failure following revision total knee arthroplasty: infection is the major cause. Int Orthop 2011;35(08):1157-1164

27 Ghomrawi HMK, Kane RL, Eberly LE, Bershadsky B, Saleh KJNorth American Knee Arthroplasty Revision (NAKAR) Study Group. Patterns of functional improvement after revision knee arthroplasty. J Bone Joint Surg Am 2009;91(12):2838-2845

28 Schroer WC, Berend KR, Lombardi AV, et al. Why are total knees failing today? Etiology of total knee revision in 2010 and 2011. J Arthroplasty 2013;28(8, Suppl):116-119 